\title{
A mouse-adapted model of SARS-CoV-2 to test COVID-19 countermeasures
}

https://doi.org/10.1038/s41586-020-2708-8

Received: 6 May 2020

Accepted: 20 August 2020

Published online: 27 August 2020

Check for updates

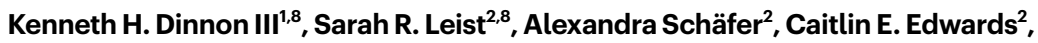 \\ David R. Martinez ${ }^{2}$, Stephanie A. Montgomery ${ }^{3}$, Ande West ${ }^{2}$, Boyd L. Yount Jr' ${ }^{2}$, Yixuan J. Hou ${ }^{2}$, \\ Lily E. Adams', Kendra L. Gully ${ }^{2}$, Ariane J. Brown ${ }^{2}$, Emily Huang ${ }^{2}$, Matthew D. Bryant ${ }^{4}$, \\ Ingrid C. Choong ${ }^{4}$, Jeffrey S. Glenn ${ }^{5,6}$, Lisa E. Gralinski ${ }^{2}$, Timothy P. Sheahan ${ }^{2}$ \& Ralph S. Baric ${ }^{1,2,7 凶}$
}

Coronaviruses are prone to transmission to new host species, as recently demonstrated by the spread to humans of severe acute respiratory syndrome coronavirus 2 (SARS-CoV-2), the causative agent of the coronavirus disease 2019 (COVID-19) pandemic ${ }^{1}$.Small animal models that recapitulate SARS-CoV-2 disease are needed urgently for rapid evaluation of medical countermeasures ${ }^{2,3}$.SARS-CoV-2 cannot infect wild-type laboratory mice owing to inefficient interactions between the viral spike protein and the mouse orthologue of the human receptor, angiotensinconverting enzyme 2 (ACE2) ${ }^{4}$. Here we used reverse genetics ${ }^{5}$ to remodel the interaction between SARS-CoV-2 spike protein and mouse ACE2 and designed mouse-adapted SARS-CoV-2 (SARS-CoV-2MA), a recombinant virus that can use mouse ACE2 for entry into cells. SARS-CoV-2 MA was able to replicate in the upper and lower airways of both young adult and aged BALB/c mice. SARS-CoV-2 MA caused more severe disease in aged mice, and exhibited more clinically relevant phenotypes than those seen in Hfh4-ACE2 transgenic mice, which express human ACE2 under the control of the $H f h 4$ (also known as Foxj1) promoter. We demonstrate the utility of this model using vaccine-challenge studies in immune-competent mice with native expression of mouse ACE2. Finally, we show that the clinical candidate interferon- $\lambda 1 \mathrm{a}$ (IFN- $\lambda 1 \mathrm{a}$ ) potently inhibits SARS-CoV-2 replication in primary human airway epithelial cells in vitro-both prophylactic and therapeutic administration of IFN- $\lambda 1 \mathrm{a}$ diminished SARS-CoV-2 replication in mice. In summary, the mouse-adapted SARS-CoV-2 MA model demonstrates age-related disease pathogenesis and supports the clinical use of pegylated IFN- $\lambda 1$ a as a treatment for human COVID-196.
Zoonotic coronaviruses are readily transmitted to new host species, as demonstrated by the emergence of SARS-CoV in 2002 and 2003, Middle East respiratory syndrome coronavirus (MERS-CoV) in 2012 and SARS-CoV-2, the causative agent of the COVID-19 pandemic. SARS-CoV-2 has caused millions of infections and hundreds of thousands of deaths worldwide. As there are currently no approved vaccines and only one antiviral agent approved ${ }^{7}$ for emergency use against SARS-CoV-2, small animal model systems are vital to improve understanding of disease mechanisms of COVID-19 and to evaluate medical countermeasures for improved global health. Mouse models can provide key insights into the pathogenic mechanisms of coronavirus disease and can serve as high-throughput preclinical evaluation platforms to identify highly performing antiviral agents and vaccines ${ }^{2,8}$. SARS-CoV-2 enters host cells by binding the cellular receptor ACE2. However, standard laboratory mice do not support infection with SARS-CoV-2 owing to incompatibility of the spike (S) protein with mouse ACE2, complicating model development ${ }^{4}$

\section{Infection of $\boldsymbol{H}$ fh 4-ACE2 transgenic mice}

Animal models will be critical for development of medical countermeasures to the COVID-19 pandemic. Laboratory mice infected with mouse-adapted strains of SARS-CoV and MERS-CoV have informed our understanding of viral pathogenesis and intervention strategies. Several animal models for SARS-CoV-2 have been reported, with varying degrees of viral replication and clinical disease, including $A C E 2$ transgenic mice ${ }^{9-11}$ and virally transduced $A C E 2$ mice $^{8,12,13}$, which express human ACE2, ferrets ${ }^{14}$, hamsters $^{15,16}$ and non-human primates $^{17-19}$. To determine the utility of mice overexpressing human ACE2 under the control of the Hfh4 (also known as Foxj1) promoter as a model for SARS-CoV-2 disease, we infected with Hfh4-ACE2 mice with SARS-CoV-2,20,21. The $H$ fh 4 promoter drives expression of human ACE2 in ciliated cells of respiratory tract epithelium and in the central nervous system ${ }^{22,23}$. Hfh4-ACE2 mice infected with SARS-CoV-2 showed little weight loss, but only $60 \%$ survived at 5 days post-infection (dpi)

'Department of Microbiology and Immunology, University of North Carolina at Chapel Hill, Chapel Hill, NC, USA. ${ }^{2}$ Department of Epidemiology, University of North Carolina at Chapel Hill,

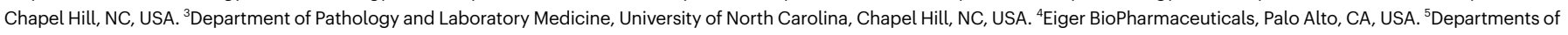
Medicine and Microbiology and Immunology, Stanford University, Stanford, CA, USA. ${ }^{6}$ Palo Alto Veterans Administration, Palo Alto, CA, USA. ${ }^{7}$ Rapidly Emerging Antiviral Drug Discovery Initiative, University of North Carolina, Chapel Hill, NC, USA. ${ }^{8}$ These authors contributed equally: Kenneth H. Dinnon III, Sarah R. Leist. ${ }^{\circledR}$ e-mail: rbaric@email.unc.edu 
(Extended Data Fig. 1a, b). Virus was detected in the lung at 2 and $5 \mathrm{dpi}$ (Extended Data Fig. 1c). Similar to previous reports of SARS-CoV and SARS-CoV-2 infection in Hfh4-ACE2 mice, SARS-CoV-2 virus was detected in the brains of mice that succumbed to infection, suggesting that mortality was driven by viral invasion of the brain ${ }^{9,21}$ (Extended Data Fig. 1d). Finally, we used whole-body plethysmography to monitor pulmonary function in mice infected with SARS-CoV- $2^{24}$. We evaluated several complementary metrics of pulmonary obstruction and bronchoconstriction, including enhanced pause $(\mathrm{PenH})$ and the fraction of expiration time at which the peak occurs (Rpef), which remained at normal levels for the duration of these studies, further indicating that respiratory infection was probably not a major driver of mortality (Extended Data Fig. 1f, g). Thus, although ACE2 expression driven by the $H f h 4$ promoter facilitates SARS-CoV-2 infection of mice, the observed pathogenesis does not accurately model the more severe disease manifestations observed in humans.

\section{Remodelling the SARS-CoV-2-ACE2 interface}

Next, instead of genetically altering the host, we sought to remodel the SARS-CoV-2S receptor-binding domain (RBD) to facilitate efficient binding to mouse ACE2. We compared the ACE2-contacting residues in the RBDs of S proteins from several group 2B coronaviruses capable of infecting mice with those of SARS-CoV-2 $21,25,26$ (Fig. 1a, Extended Data Fig. 2). There were variations in this region in $\mathrm{S}$ proteins from SARS-CoV, WIV1 and SHC014 at a few amino acid positions, but residue 498 of SARS-CoV-2 was uniquely divergent, suggesting incompatibility of SARS-CoV-2 Q498 with mouse ACE2. In addition, molecular modelling of the SARS-CoV-2 S RBD-receptor interface predicted a loss of the interaction between Q498 of SARS-CoV-2 S protein and Q42 of human ACE2 with mouse ACE2 (Fig. 1b, c), which may diminish binding efficiency. Thus, substitution of residue Q498 and the adjacent P499 with $Y$ and T, respectively, from WIV1 and SARS-CoV might restore the interaction with Q42 of mouse ACE2 while preserving the interaction with human ACE2 (Fig. 1d, e). Using reverse genetics ${ }^{22}$, we engineered Q498Y/P499T into the SARS-CoV-2S gene and recovered the recombinant virus (SARS-CoV-2 MA). Notably, SARS-CoV-2 MA replicated with slightly lower titres compared with parental wild-type virus in Vero E6 cells (Fig. 1f) and primary differentiated bronchiolar human airway epithelial (HAE) cells (Fig. 1g). In contrast to wild-type SARS-CoV-2, SARS-CoV-2 MA RNA could be detected in cells expressing mouse ACE2 by $24 \mathrm{~h}$ after infection (Fig. $1 \mathrm{~h}$ ).

\section{SARS-CoV-2 MA replicates in mouse airways}

After demonstrating SARS-CoV-2 MA could use mouse ACE2 for entry into cells, we sought to determine whether this virus could infect young adult wild-type mice. Although overt clinical signs of infection such as weight loss were not observed in young adult BALB/c mice infected with $10^{5}$ plaque-forming units (PFU) SARS-CoV-2 MA (Fig. 2a), high-titre virus replication was observed in lung tissue at $2 \mathrm{dpi}$, but was cleared by $4 \mathrm{dpi}$ (Fig. 2b). Under identical conditions, replication of wild-type SARS-CoV-2 was not detected. Using whole-body plethysmography, we found that young adult mice infected with SARS-CoV-2 MA showed a small but significant change in PenH (Fig. 2d) and a significant decrease in Rpef (Fig. 2e) at 2 dpi, indicative of impaired lung function. Histological analysis of mice infected with SARS-CoV-2 MA revealed insterstitial congestion, epithelial damage, inflammatory infiltrate and peribronchiolar lympocytic inflammation surrounding airways at $2 \mathrm{dpi}$; viral antigen staining revealed these to be the main sites of viral replication (Fig. 2f). At 4 dpi, histological analysis showed increased inflammation and haemorrhage in the lung (Fig. 2g). Concordant with infectious titre data demonstrating virus clearance by $4 \mathrm{dpi}$, viral antigen was not detected in lung tissue sections at this time (Fig. 2b, g). Similar to observations of SARS-CoV-2 in humans, SARS-CoV-2 MA was observed in the upper airway and viral antigen was present in nasal turbinate epithelium at 2 dpi (Fig. 2c, h). Thus, similar to SARS-CoV-2 infection in humans, SARS-CoV-2 MA infection in young adult mice resulted in efficient virus replication in the upper and lower airways and limited replication in the parenchyma, and was associated with mild-to-moderate disease.

\section{Age effect on SARS-CoV-2 disease in mice}

Higher morbidity and mortality rates have been consistently observed in older human populations throughout the COVID-19 pandemic ${ }^{27}$. Additionally, wild-type and mouse-adapted SARS-CoV show highly age-dependent disease phenotypes in humans and mice, respectively ${ }^{28,29}$. To determine whether the infection of aged mice with SARS-CoV-2 MA would recapitulate the age-dependent increase in disease severity observed in humans with COVID-19, we infected one-year-old BALB/c mice with SARS-CoV-2 MA. In contrast to young adult mice, aged BALB/c mice exhibited a transient but significant decrease in body weight at 3-4 dpi compared with mock-infected mice (Fig. 3a) (old versus young, $P<0.0001$ ( $3 \mathrm{dpi}$ ) and $P<0.0040$ $(4 \mathrm{dpi}))$. Similar to young adult mice, aged mice had high viral titre in the lung at $2 \mathrm{dpi}$, but in contrast to young adult mice, viral clearance in the aged mice was delayed, as indicated by detectable virus at $4 \mathrm{dpi}$ (Fig. 3b). Similarly, replication in the upper airway persisted in half of the aged mice at $4 \mathrm{dpi}$ (Fig. 3c). The loss of pulmonary function was more pronounced in aged animals, as shown by significant differences in PenH and Rpef among mock-infected and SARS-CoV-2 MA-infected mice (Fig. 3d, e). PenH was significantly higher in aged mice infected with SARS-CoV-2 MA at 2 dpi compared with young mice infected with SARS-CoV-2 MA $(P=0.0457)$. Rpef was significantly lower in aged infected mice at $2 \mathrm{dpi}(P=0.0264)$ and $4 \mathrm{dpi}(P=0.0280)$. Compared to young mice, aged mice infected with SARS-CoV-2 MA displayed increased epithelial damage, peribronchiolar lymphocytic inflammation, haemorrhage and oedema in the lung at $2 \mathrm{dpi}$ and $4 \mathrm{dpi}$, and viral antigen was found in conducting airway epithelium, interstitium and nasal epithelium, with minimal antigen staining at $4 \mathrm{dpi}$, concordant with detection of viral titre (Fig. 3b, f-h). Additionally, levels of several proinflammatory cytokines were increased in the lung but not in the serum at $2 \mathrm{dpi}$, indicative of a localized cytokine and chemokine response (Extended Data Fig. 3).

\section{Vectored vaccine and IFN- $\boldsymbol{\lambda l a}$ efficacy}

As demonstrated with mouse-adapted strains of SARS-CoV ${ }^{25}$, the replication-competent SARS-CoV-2 MA strain facilitates the identification of virus and host factors that guide pathogenesis and disease severity and enables rapid testing of intervention strategies in standard laboratory mice. Using a virus replicon particle (VRP) system, we vaccinated ten-week-old BALB/c mice against SARS-CoV-2 S and nucleocapsid (N), with GFP as a control, with a boost after three weeks, and challenged them four weeks after the boost with SARS-CoV-2 MA. Three weeks after the boost, serum from mice vaccinated with $S-$ but not from mice vaccinated with GFP or N-potently neutralized SARS-CoV-2 reporter virus expressing nanoluciferase (nLUC) (Fig. 4a). Upon challenge with SARS-CoV-2 MA, only mice vaccinated with VRP expressing S exhibited significantly diminished viral titre in the lungs and nasal turbinate (Fig. 4b, c).

IFN- $\lambda$ is a type III interferon; its receptors are largely limited to epithelial cells, including those in the lungs ${ }^{30,31}$. Treatment with IFNs has been used as panviral treatment for several viral infections, including in trials for the treatment of SARS-CoV and MERS-CoV infections. Pegylated human interferon lambda-1 (PEG-IFN- $\lambda 1$ ) is a phase-3-ready treatment for hepatitis delta virus infection and has been proposed as a treatment for patients with COVID-1932. Thus, we sought to determine whether PEG-IFN- $\lambda 1$ would initiate an antiviral response capable of inhibiting productive infection of HAE cell cultures by SARS-CoV-2. Pretreatment 


\begin{tabular}{|c|c|c|c|c|c|c|c|c|c|c|c|c|c|c|c|c|c|c|c|}
\hline & 402 & 426 & 436 & 440 & 442 & 443 & 462 & 463 & 472 & 473 & 475 & 479 & 482 & 484 & 486 & 487 & 488 & 489 & 491 \\
\hline & $\mathbf{T}$ & $\mathbf{R}$ & $\mathbf{Y}$ & $\bar{Y}$ & $\mathbf{Y}$ & $\mathbf{L}$ & $s$ & D & $\mathbf{L}$ & $\mathbf{N}$ & $\bar{Y}$ & $\mathbf{N}$ & $\mathbf{G}$ & $\mathbf{Y}$ & $T$ & $T$ & $\mathbf{G}$ & I & $\mathbf{Y}$ \\
\hline & $T$ & $\mathbf{R}$ & $\mathbf{H}$ & $\mathbf{Y}$ & $Y$ & 1 & $\mathbf{S}$ & D & $\mathbf{L}$ & $\mathbf{N}$ & $\mathbf{Y}$ & $\mathbf{N}$ & & $\mathbf{Y}$ & 1 & $T$ & $G$ & & $\mathbf{Y}$ \\
\hline & $T$ & $\mathbf{R}$ & $Y$ & $Y$ & s & L & $\mathbf{s}$ & [ & F & $N$ & $\bar{Y}$ & $\mathbf{N}$ & & $\mathbf{Y}$ & $\mathbf{T}$ & $\mathbf{N}$ & & & $\mathbf{Y}$ \\
\hline 074 & $\bar{T}$ & $\mathbf{N}$ & $Y$ & $\mathbf{Y}$ & W & $\mathbf{v}$ & $\mathbf{s}$ & $\mathbf{G}$ & & 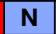 & $\mathbf{Y}$ & $\mathbf{R}$ & & $\mathbf{F}$ & 1 & A & & $\mathbf{v}$ & $\mathbf{H}$ \\
\hline \multirow[t]{2}{*}{ ARS-CoV-2 } & $\mathbf{T}$ & $\mathbf{N}$ & $\mathbf{Y}$ & $\mathbf{Y}$ & $\mathbf{L}$ & $\mathbf{F}$ & $\mathbf{M}$ & G & 8 & iv & Y & 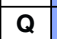 & G & $\mathbf{Q}$ & 1 & $\mathbf{N}$ & G & $\mathbf{v}$ & $\mathbf{Y}$ \\
\hline & 415 & 439 & 449 & $\mid 453$ & 455 & 456 & 475 & 476 & 4866 & 487 & 489 & 493 & 496 & 498 & 500 & 501 & 502 & 503 & 50 \\
\hline
\end{tabular}

b

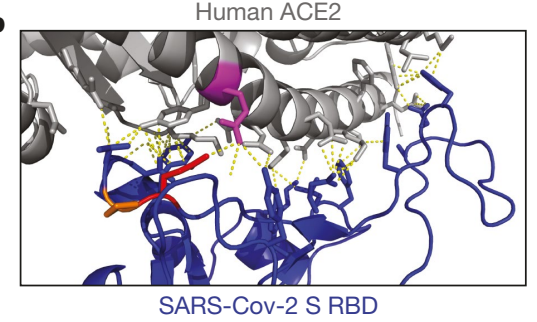

d

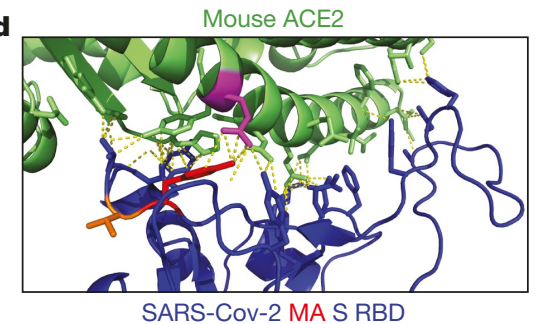

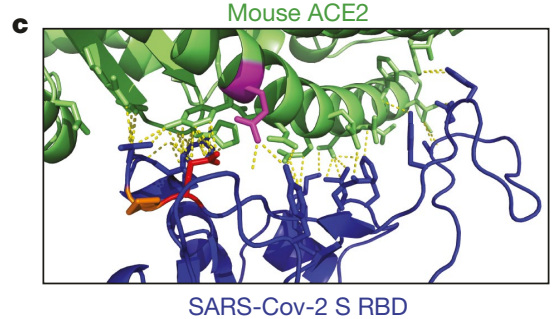

e

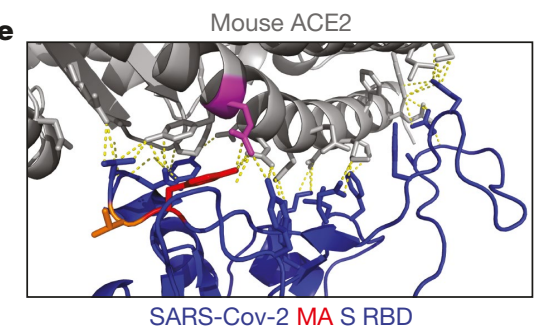

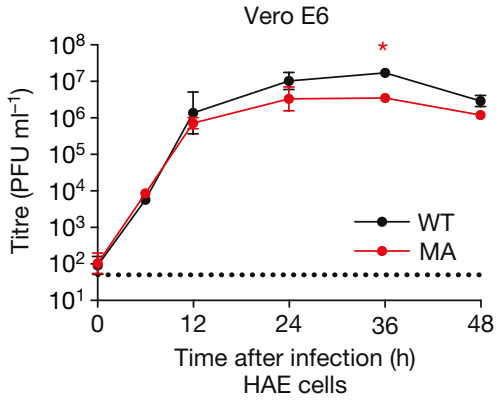

$\mathbf{g}$

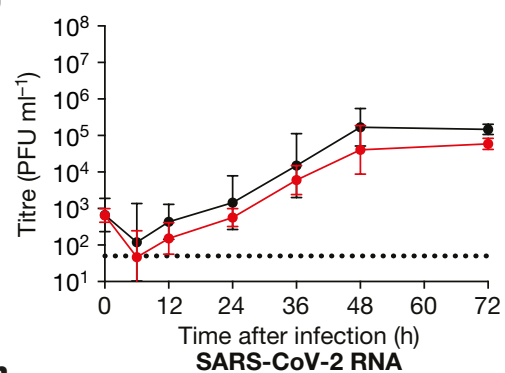

h

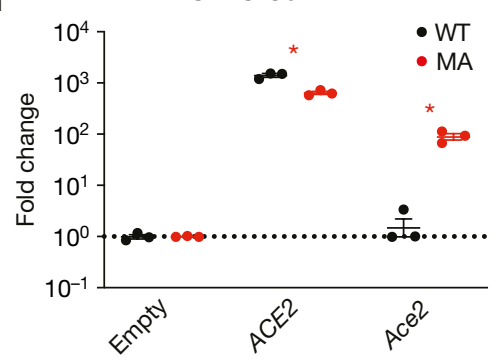

Fig. 1 | Generation of SARS-CoV-2 MA. a, Table of group 2B S RBD-ACE2 interacting residues. Amino acid positions are numbered relative to SARS-CoV (top row) and SARS-CoV-2 (bottom row). Green shading indicates contacts as determined by published crystal structures. Amino acids are coloured by BLOSUM62 conservation score relative to S protein from SARS-CoV Urbani (red, least conserved; blue, most conserved). SARS-CoV Urbani, SARS-CoV MA15, WIV1 and SHC014 S proteins can use mouse ACE2 as a functional receptor, whereas SARS-CoV-2S cannot. The red outline indicates Q498 in SARS-CoV-2S, which makes contact with human ACE2 in both SARS-CoV Sand SARS-CoV-2S, and is divergent in SARS-CoV-2.b, SARS-CoV-2S RBD-human ACE2 interface (PDB: 6MOJ). SARS-CoV-2S Q498 (red) interacts with Q42 (magenta) of human ACE2.c, Modelling of SARS-CoV-2S RBD and mouse ACE2. SARS-CoV-2S Q498 does not interact with Q42 of mouse ACE2. d, Modelling of SARS-CoV-2S(Q498Y/P499T) (orange) shows restored interaction with Q42 of mouse ACE2.e, Modelling of SARS-CoV-2S(Q498Y/P499T) showing interaction with Q42 of human ACE2.f, g, Single-step growth curve of SARS-CoV-2 (WT) and SARS-CoV-2 MA (MA) in Vero E6 (f) and HAE (g) cells. The dotted line represents LOD. The log-transformed data were analysed by two-way analysis of variance (ANOVA) followed by Sidak's multiple comparisons. Inf, ${ }^{*} P=0.0053(36 \mathrm{~h})$. h, Non-permissive DBT-9 cells were transfected to express human $A C E 2$ or mouse $A c e 2$ and infected with SARS-CoV-2 or SARS-CoV-2 MA. Viral RNA was quantified by quantitative PCR with reverse transcription (RT-qPCR) $24 \mathrm{~h}$ after infection and normalized expression in cells transfected with empty vector. The log-transformed data were analysed by two-way ANOVA followed by Dunnett's multiple comparisons. ${ }^{*} P=0.0322$ (ACE2), ${ }^{*} P<0.0001$ (Ace2). In $\mathbf{f}-\mathbf{h}, n=3$ technical replicates for each group, representative of 2 independent experiments. Data are mean \pm s.d.

these data demonstrate the utility of these models for rapid evaluation of vaccines and therapeutic drug efficacy in standard laboratory mice. In addition, they demonstrate that PEG-IFN- $\lambda 1$ exerts potent antiviral activity against SARS-CoV- 2 in vitro and can diminish virus replication in vivo even when given therapeutically.

\section{Discussion}

Structural studies have identified the residues in SARS-CoV and SARS-CoV-2 S-protein RBD that bind human ACE2 ${ }^{33,34}$. Using molecular modelling and reverse genetics ${ }^{22}$, we altered the SARS-CoV-2 RBD to enable viral entry via mouse ACE2, highlighting the precision of the structure-based predictions. Unlike parental wild-type virus, the resultant recombinant virus (SARS-CoV-2 MA) is able to use mouse ACE2 to infect cells in vitro. Recombinant SARS-CoV-2 MA did not grow more efficiently than wild-type virus in primary HAE or Vero cells, suggesting that the fitness of the virus is not increased in human cells. SARS-CoV-2 MA replicated in both the upper and 

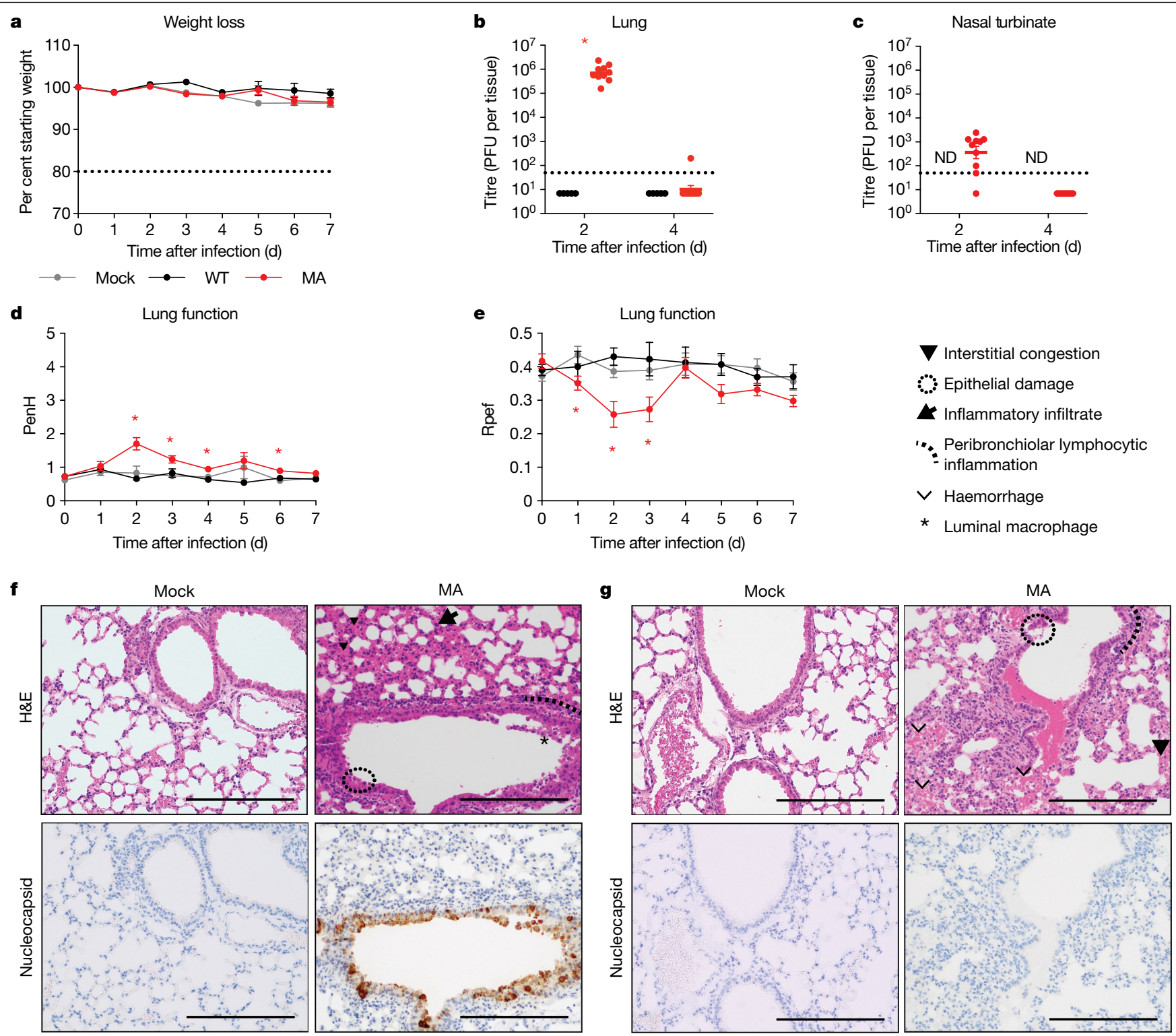

h

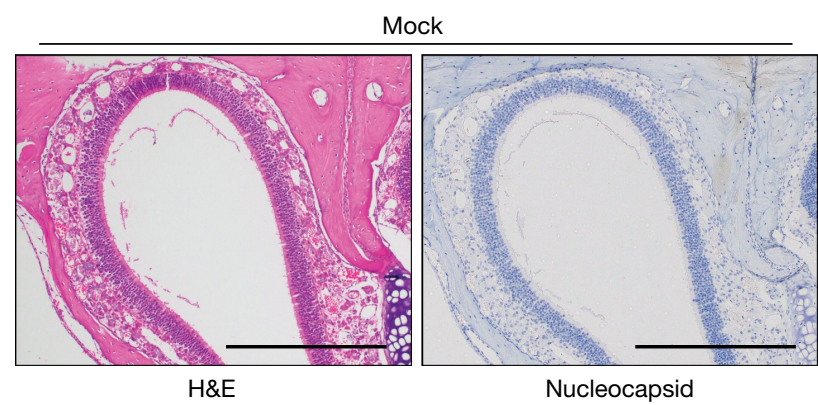

Fig. 2 |SARS-CoV-2 MA replicates in young BALB/c mice. a-h, Twelve-weekold female BALB/c mice were mock-infected (grey) (a,d,e) or infected with $10^{5}$ PFU wild-type SARS-CoV-2 (black) or SARS-CoV-2 MA (red) (a-e). Combined data from two independent experiments. a, Weight loss. The dotted line represents weight-loss criteria for humane euthanasia. Data were analysed using mixed-effects analysis followed by Dunnett's multiple comparisons. b, c, Viral lung (b) and nasal turbinate (c) titre (2 dpi: $n=5$ (WT), $n=10$ (MA); $4 \mathrm{dpi}: n=5(\mathrm{WT}), n=9(\mathrm{MA}))$. The dotted line represents LOD. Undetected samples are plotted at half the LOD. The log-transformed data were analysed by two-way ANOVA followed by Sidak's multiple comparisons. ND, not

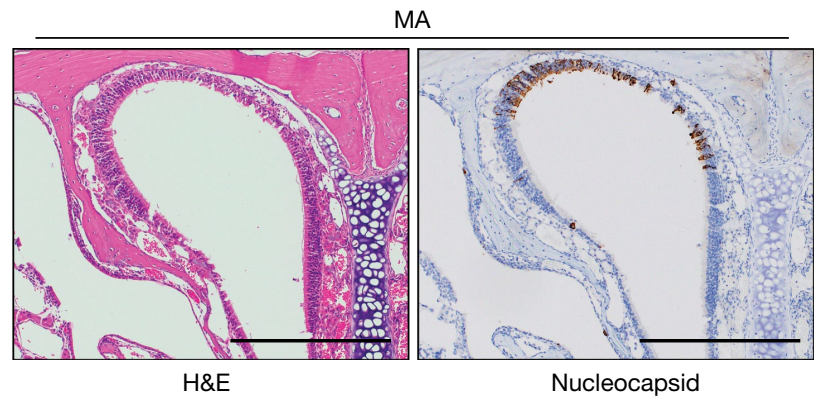

determined. In $\mathbf{b},{ }^{*} P \leq 0.0001$. d, e, Whole-body plethysmography assessing pulmonary function for PenH (d) and Rpef (e). Data were analysed by two-way ANOVA followed by Dunnett's multiple comparisons. Data are mean \pm s.e.m. In d, ${ }^{*} P=0.012$ ( $\left.2 \mathrm{dpi}\right), 0.0025$ (3 dpi), 0.0030 (4 dpi), 0.0018 (6 dpi). In e, ${ }^{*} P=0.0426$ (1 dpi), 0.0194 ( $\left.2 \mathrm{dpi}\right), 0.0442$ ( $\left.3 \mathrm{dpi}\right) . \mathbf{f}-\mathbf{h}$, Lung sections at $2 \mathrm{dpi}(\mathbf{f})$ and $4 \mathrm{dpi}$ (g), and nasal turbinates at $2 \mathrm{dpi}$ (h). Haematoxylin and eosin (H\&E) staining and immunohistochemistry staining for SARS-CoV-2 $\mathrm{N}$ protein, counterstained with haematoxylin. Scale bars, $200 \mu \mathrm{m}$. Images representative of two independent experiments. 


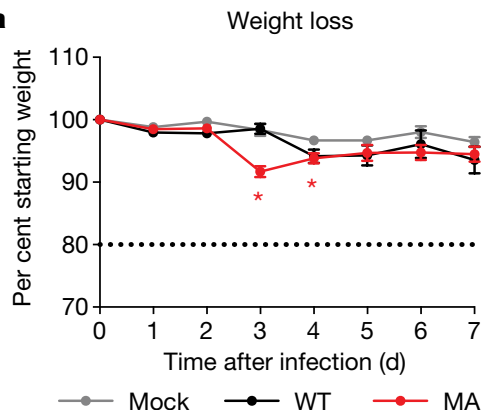

d

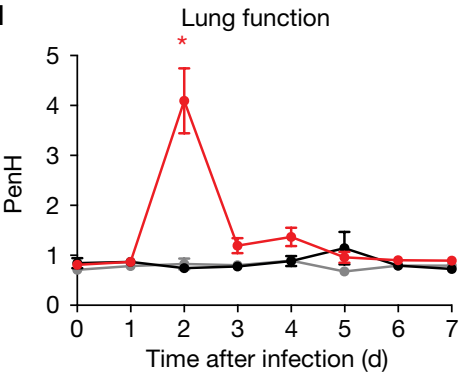

b

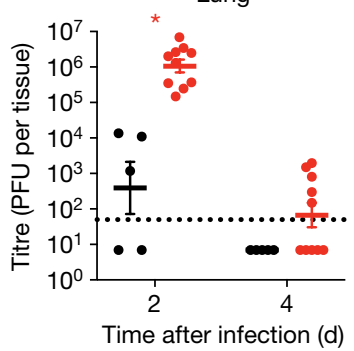

e

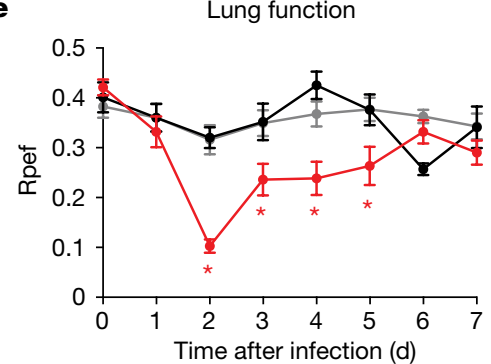

c

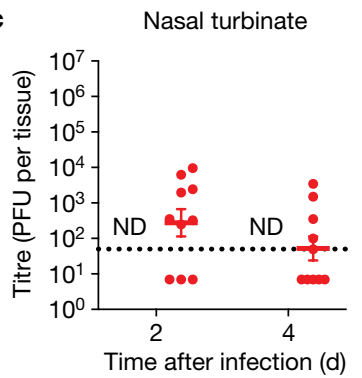

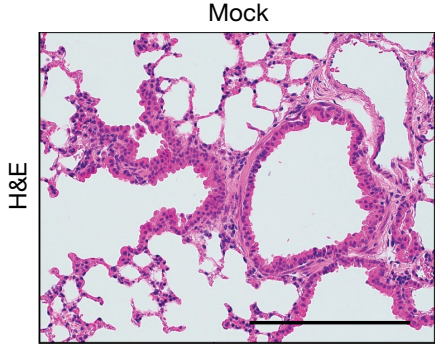

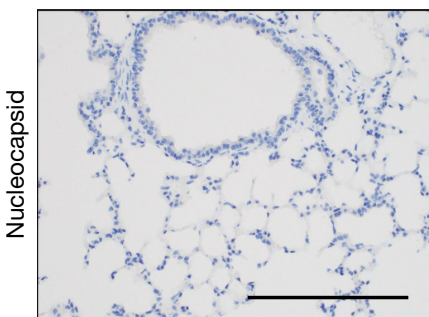

h

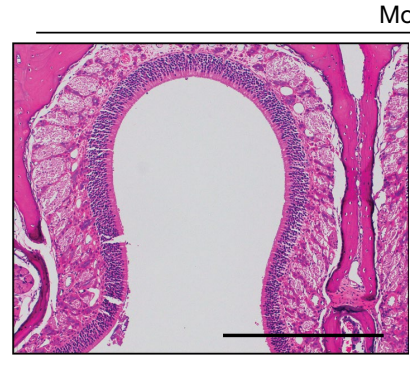

H\&E

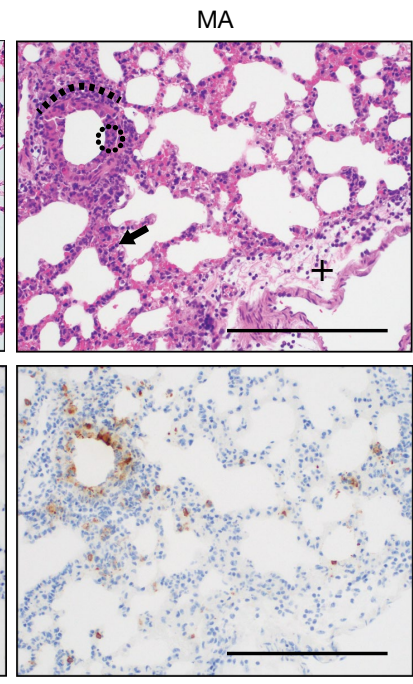

Mock

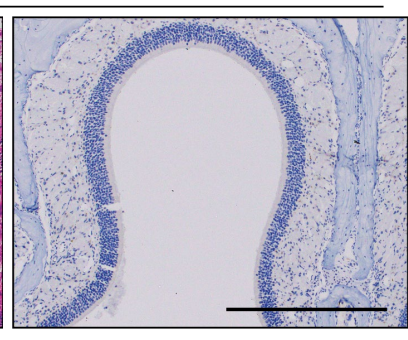

Nucleocapsid

Fig. 3 | SARS-CoV-2 MA replicates in old BALB/c mice with minor disease. a-h, One-year-old female BALB/c were mock-infected (grey) or infected with $10^{5}$ PFU SARS-CoV-2 (black) or SARS-CoV-2 MA (red). Combined data from two independent experiments. a, Weight loss. The dotted line represents weight-loss criteria for humane euthanasia. Data were analysed by mixed-effects analysis followed by Dunnett's multiple comparisons. ${ }^{*} P \leq 0.0001$ ( $\left.3 \mathrm{dpi}\right), 0.0305$ (4 dpi). b, c, Viral lung $(\mathbf{b})$ and nasal turbinate (c) titres. $n=5(\mathrm{WT}), n=10(\mathrm{MA})$. The dotted line represents LOD. Undetected samples are plotted at half the LOD. The log-transformed data were analysed by

lower airways of BALB/c mice, and resulted in more severe disease in aged mice, reproducing the age-related increase in disease severity observed in humans. SARS-CoV-2 MA infection is cleared by $4 \mathrm{dpi}$ in
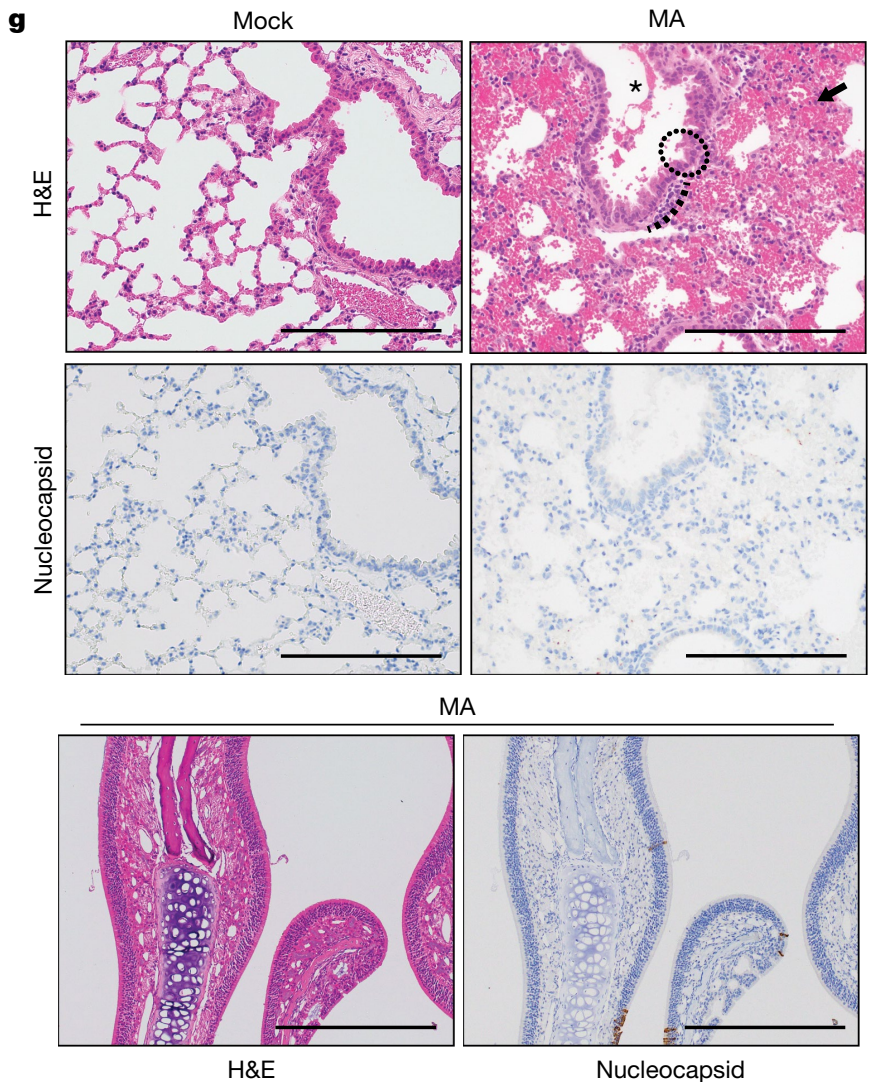

two-way ANOVA followed by Sidak's multiple comparisons. In $\mathbf{b},{ }^{*} P \leq 0.0001$. d, e, Whole-body plethysmography assessing pulmonary function for $\mathrm{PenH}$ (d) and Rpef (e). Data were analysed by two-way ANOVA followed by Dunnett's multiple comparisons. Data are mean \pm s.e.m. In d, ${ }^{*} P=0.0014$ ( $\left.2 \mathrm{dpi}\right)$. In e, ${ }^{*} P \leq 0.0001$ ( $2 \mathrm{dpi}$ ), 0.0242 ( $3 \mathrm{dpi}$ ), 0.0130 (4 dpi), 0.0481 ( $5 \mathrm{dpi}$ ).f-h, Lung sections at $2 \mathrm{dpi}(\mathbf{f})$ and $4 \mathrm{dpi}(\mathbf{g})$, and nasal turbinates at $2 \mathrm{dpi}(\mathbf{h})$. H\&E staining and immunohistochemistry staining for SARS-CoV-2 N protein, counterstained with haematoxylin. Scale bars, $200 \mu \mathrm{m}$. Images representative of two independent experiments.

young but not in aged adult mice, probably owing to control by IFNs and the innate immune system ${ }^{12,35}$, and is exemplified by sensitivity to PEG-IFN- $\lambda 1$. 


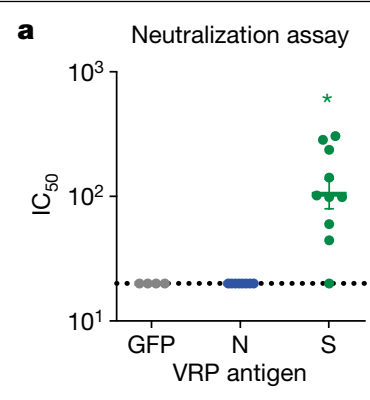

d
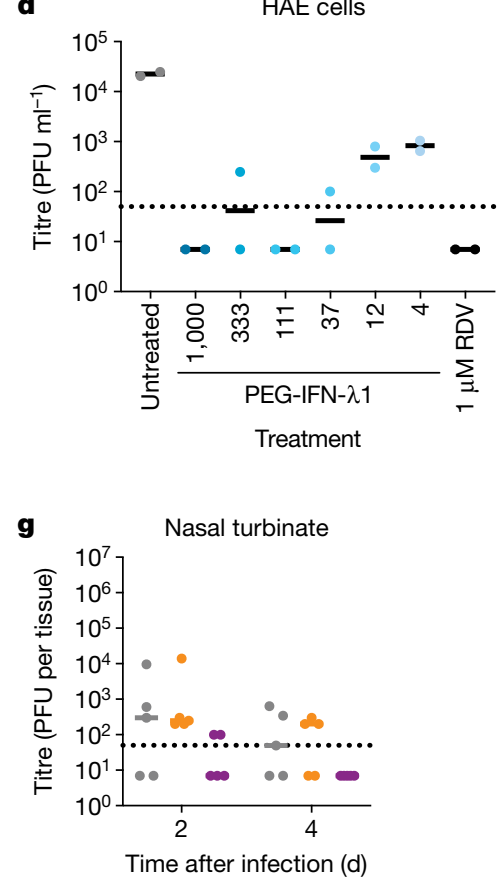

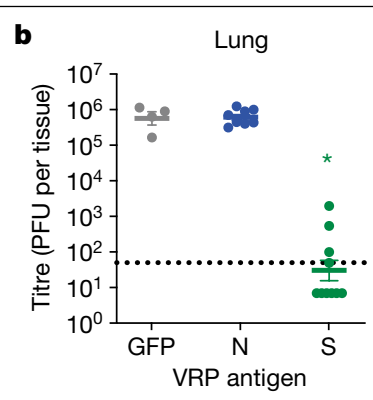

e

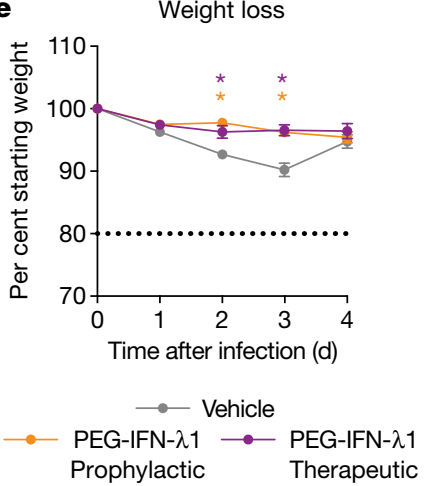

h

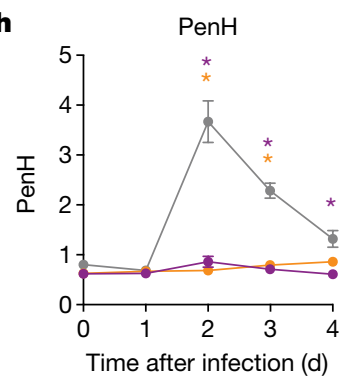

c

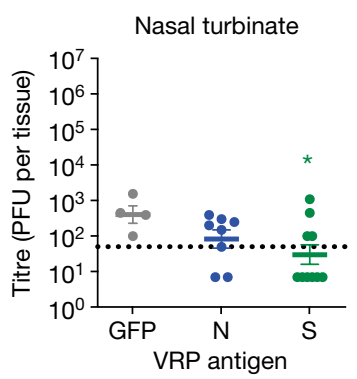

f

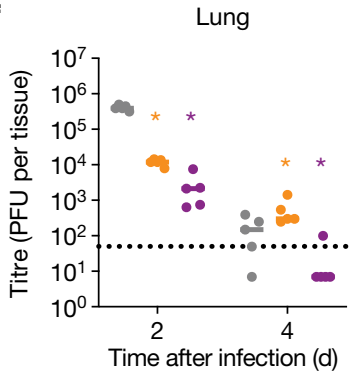

i

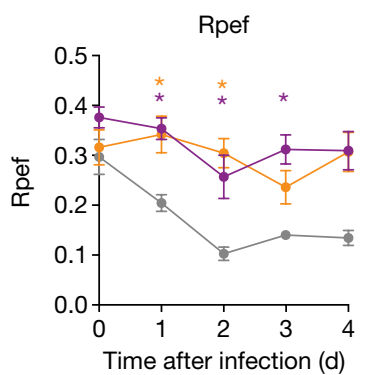

Fig. 4 | Evaluation of prevention and intervention strategies against SARS-CoV-2 MA infection in mice. a-c, Groups of 10-week-old female BALB/c mice were vaccinated with VRPs expressing wild-type $\mathrm{S}(n=10), \mathrm{N}(n=8)$ or GFP $(n=4)$. a, Half-maximal inhibitory concentration $\left(\mathrm{IC}_{50}\right)$ of sera taken three weeks after boost to neutralize SARS-CoV-2. The dotted line represents LOD. The log-transformed data were analysed by one-way ANOVA followed by Dunnett's multiple comparisons. ${ }^{*} P=0.0003 . \mathbf{b}, \mathbf{c}$, Lung (b) and nasal turbinate (c) viral titre at $2 \mathrm{dpi}$. The dotted line represents LOD. Undetected samples are plotted at half the LOD. The log-transformed data were analysed as in $\mathbf{a}$. In $\mathbf{b}$, ${ }^{*} P \leq 0.0001$. In $\mathbf{c},{ }^{*} P=0.0360$. d, Human primary airway epithelial cells were pretreated for $24 \mathrm{~h}$ with PEG-IFN- $\lambda 1$, followed by infection with SARS-CoV-2. Infectious virus in apical washes from $48 \mathrm{~h}$ after infection was titrated. Remdesivir (RDV) was used as positive control. Data are representative of two independent experiments with samples from distinct human donors. e-i, One-year-old female BALB/c mice were subcutaneously treated with vehicle or with $2 \mu \mathrm{g}$ PEG-IFN- $\lambda 1$ prophylactically or therapeutically and infected with SARS-CoV-2 MA. $n=5$ per group per time point. e, Weight loss. The dotted line represents weight-loss criteria for humane euthanasia. Data were analysed by mixed-effects analysis followed by Dunnett's multiple comparisons. In $\mathbf{b},{ }^{*} P \leq 0.0001$ (prophylactic, $2 \mathrm{dpi}$ ), 0.0128 (therapeutic, $2 \mathrm{dpi}$ ), 0.0042 (prophylactic, $4 \mathrm{dpi}$ ), 0.0037 (therapeutic, $4 \mathrm{dpi}$ ).f, g, Viral titres in the lung (f) and nasal turbinates (g) at 2 and $4 \mathrm{dpi}$. The dotted line represents LOD. The log-transformed data were analysed by two-way ANOVA followed by Dunnett's multiple comparisons. $\mathbf{h}, \mathbf{i}$, Whole-body plethysmography assessing pulmonary function for PenH (h) and Rpef (i). Data were analysed as in e. Data are mean \pm s.e.m. In $\mathbf{h},{ }^{*} P=0.0083$ (prophylactic, $2 \mathrm{dpi}$ ), 0.0080 (therapeutic, $2 \mathrm{dpi}$ ), 0.0029 (prophylactic, $3 \mathrm{dpi}$ ), 0.0020 (therapeutic, $3 \mathrm{dpi}$ ), 0.0327 (therapeutic, $4 \mathrm{dpi}$ ). In $\mathbf{i},{ }^{*} P=0.0442$ (prophylactic, $1 \mathrm{dpi}$ ), 0.0033 (therapeutic, $1 \mathrm{dpi}), 0.0048$ (prophylactic, $2 \mathrm{dpi}$ ), 0.0118 (therapeutic, $3 \mathrm{dpi}$ ), 0.0259 (prophylactic, $4 \mathrm{dpi}$ ), 0.0247 (therapeutic, $4 \mathrm{dpi}$ ).
SARS-CoV-2 is a pandemic pathogen of concern and the risk potential of experiments to intentionally alter host range was reviewed for potential pandemic pathogen care and oversight (P3C0) concerns before the research was started. By current US review standards, SARS-CoV-2 MA was not considered dual-use research of concern, which is limited to a subset of pathogens. Before the start of the studies, appropriate discussion and documentation of proposed experiments and protocols were reviewed and approvals were obtained by institutional and external review. We recommend that SARS-CoV-2 MA and its derivitives are maintained in a biosafety level 3 laboratory.

We show the utility of the SARS-CoV-2 MA model for screening medical countermeasures through vaccine challenge studies and the evaluation of a clinical candidate, PEG-IFN- $\lambda 1$. PEG-IFN- $\lambda 1$ is a phase-3-ready drug in clinical development for hepatitis delta virus infection. It has been given to more than 3,000 patients in the context of 19 clinical trials as a weekly subcutaneous injection, often for 24-48 weeks, to treat patients with chronic viral hepatitis ${ }^{36,37}$. PEG-IFN- $\lambda 1$ is a promising therapy for the treatment of SARS-CoV-2 infection ${ }^{6}$, and blocks porcine coronavirus replication in gut cells in vitro ${ }^{38}$ and SARS-CoV replication in human airway cells ${ }^{39}$. Our results, which demonstrate reduction in SARS-CoV-2 infection in primary human cells and in mice, support further multiple-investigator sponsored studies currently underway to evaluate PEG-IFN- $\lambda 1$ for prevention and treatment of SARS-CoV-2 infection (Clinicaltrials. gov identifiers: NCT04331899, NCT04343976, NCT04344600 and NCT04354259). 
Although the mutations in the SARS-CoV-2 MA S-protein RBD may attenuate the function of some human monoclonal antibodies or vaccines in mice, this phenotype was not observed with mouse-adapted strains of SARS- $\mathrm{CoV}^{40-42}$ and the mutations did not alter the potent neutralizing activity of two human monoclonal antibodies that target the SARS-CoV-2S-protein $\mathrm{RBD}^{43}$. We also demonstrate that the $H$ fh $4-A C E 2$ transgenic mouse model ${ }^{21}$ supports efficient SARS-CoV-2 replication and pathogenesis in vivo. Thus, this transgenic mouse model offers an alternative model of replication and disease that uses wild-type SARS-CoV-2 and is appropriate for evaluating therapeutic antibodies and other countermeasures that target RBD epitopes that are altered in SARS-CoV-2 MA.

Similar to SARS-CoV infection, SARS-CoV-2 infection of Hfh4-ACE2 transgenic mice results in mild bronchiolitis in young mice and about $40 \%$ mortality; this mortality is associated with viral invasion of the central nervous system ${ }^{9,21,44}$. A recent study used a mouse $A c e 2$ promoter to drive overexpression of $A C E 2$, and infected 6-to11-month-old mice, resulting in mild weight loss and pulmonary inflammation ${ }^{10}$. The discrepancy in weight loss between that study and the Hfh4-ACE2 model presented here may be a result of differences in the age of mice used or differences in distribution of $A C E 2$ expression. Models using viral delivery to overexpress human ACE2 in mice have also been reported ${ }^{8,12}$. Although these systems allow for rapid studies in commercially available mice, including knockout mice, the cellular distribution of human ACE2 may not faithfully recapitulate endogenously expressed ACE2 and proper SARS-CoV-2 tropism.

Our SARS-CoV-2 MA model captures multiple aspects of SARS-CoV-2 pathogenesis in young and aged BALB/c mice and provides an urgently needed high-throughput in vivo system to evaluate medical countermeasures during this devastating pandemic. As the model uses standard immune-competent laboratory mice, its accessibility, ease of use, availability of reagents, cost and utility are more favourable than for other $A C E 2$ transgenic mice, ferret, hamster and non-human primate models. The model also provides a key first step in the serial adaptation of SARS-CoV-2 in mice ${ }^{25}$, which could potentially select for variants that develop more severe pathogenic manifestations of acute respiratory distress syndrome, coagulopathies and other human disease outcomes. In addition, the SARS-CoV-2 MA model can be used to evaluate the role of host genetics and antiviral defence genes in viral pathogenesis using transgenic and knockout mice. Together, these data describe two new animal models with distinct features for testing of different medical countermeasures.

\section{Online content}

Any methods, additional references, Nature Research reporting summaries, source data, extended data, supplementary information, acknowledgements, peer review information; details of author contributions and competing interests; and statements of data and code availability are available at https://doi.org/10.1038/s41586-020-2708-8.

1. Li, Q. et al. Early transmission dynamics in Wuhan, China, of novel coronavirus-infected pneumonia. N. Engl. J. Med. 382, 1199-1207 (2020).

2. Corbett, K. S. et al. SARS-CoV-2 mRNA vaccine development enabled by prototype pathogen preparedness. Nature https://doi.org/10.1038/s41586-020-2622-0 (2020).

3. Hassan, A. O. et al. A SARS-CoV-2 infection model in mice demonstrates protection by neutralizing antibodies. Cell. 182, 744-753 (2020).

4. Zhou, P. et al. A pneumonia outbreak associated with a new coronavirus of probable bat origin. Nature 579, 270-273 (2020).

5. Hou, Y. J. et al. SARS-CoV-2 reverse genetics reveals a variable infection gradient in the respiratory tract. Cell 182, 429-446 (2020).

6. Andreakos, E. \& Tsiodras, S. COVID-19: lambda interferon against viral load and hyperinflammation. EMBO Mol. Med. 12, e12465 (2020).

7. Humeniuk, R. et al. Safety, tolerability, and pharmacokinetics of remdesivir, an antiviral for treatment of COVID-19, in healthy subjects. Clin. Transl. Sci. 13, 896-906 (2020).
8. Hassan, A. O. et al. A SARS-CoV-2 infection model in mice demonstrates protection by neutralizing antibodies. Cell 182, 744-753 (2020).

9. Jiang, R. D. et al. Pathogenesis of SARS-CoV-2 in transgenic mice expressing human angiotensin-converting enzyme 2. Cell 182, 50-58 (2020).

10. Bao, L. et al. The pathogenicity of SARS-CoV-2 in hACE2 transgenic mice. Nature $\mathbf{5 8 3}$ 830-833 (2020)

11. Sun, S. H. et al. A mouse model of SARS-CoV-2 infection and pathogenesis. Cell Host Microbe 28, 124-133 (2020).

12. Sun, J. et al. Generation of a broadly useful model for COVID-19 pathogenesis, vaccination, and treatment. Cell 182, 734-743 (2020).

13. Israelow, B. et al. Mouse model of SARS-CoV-2 reveals inflammatory role of type I interferon signaling. J. Exp. Med. 217, e20201241 (2020).

14. Kim, Y. I. et al. Infection and rapid transmission of SARS-CoV-2 in ferrets. Cell Host Microbe 27, 704-709 (2020).

15. Imai, M. et al. Syrian hamsters as a small animal model for SARS-CoV-2 infection and countermeasure development. Proc. Natl Acad. Sci. USA 117, 16587-16595 (2020).

16. Sia, S. F. et al. Pathogenesis and transmission of SARS-CoV-2 in golden hamsters. Nature 583, 834-838 (2020).

17. Rockx, B. et al. Comparative pathogenesis of COVID-19, MERS, and SARS in a nonhuman primate model. Science 368, 1012-1015 (2020).

18. Yu, P. et al. Age-related rhesus macaque models of COVID-19. Animal Model. Exp. Med. 3, 93-97 (2020).

19. Munster, V. J. et al. Respiratory disease in rhesus macaques inoculated with SARS-CoV-2. Nature 585, 268-272 (2020).

20. Ostrowski, L. E., Hutchins, J. R., Zakel, K. \& O’Neal, W. K. Targeting expression of a transgene to the airway surface epithelium using a ciliated cell-specific promoter. Mol. Ther. 8, 637-645 (2003).

21. Menachery, V. D. et al. SARS-like WIV1-CoV poised for human emergence. Proc. Natl Acad. Sci. USA 113, 3048-3053 (2016).

22. Hou, Y. J. et al. SARS-CoV-2 reverse genetics reveals a variable infection gradient in the respiratory tract. Cell 182, 429-446 (2020).

23. Abdi, K. et al. Uncovering inherent cellular plasticity of multiciliated ependyma leading to ventricular wall transformation and hydrocephalus. Nat. Commun. 9, 1655 (2018).

24. Menachery, V. D., Gralinski, L. E., Baric, R. S. \& Ferris, M. T. New metrics for evaluating viral respiratory pathogenesis. PLOS ONE 10, e0131451 (2015).

25. Roberts, A. et al. A mouse-adapted SARS-coronavirus causes disease and mortality in BALB/c mice. PLoS Pathog. 3, e5 (2007).

26. Menachery, V. D. et al. A SARS-like cluster of circulating bat coronaviruses shows potential for human emergence. Nat. Med. 21, 1508-1513 (2015).

27. Wang, D. et al. Clinical characteristics of 138 hospitalized patients with 2019 novel coronavirus-infected pneumonia in Wuhan, China. J. Am. Med. Assoc. (2020).

28. de Wit, E., van Doremalen, N., Falzarano, D. \& Munster, V. J. SARS and MERS: recent insights into emerging coronaviruses. Nat. Rev. Microbiol. 14, 523-534 (2016).

29. Roberts, A. et al. Aged BALB/c mice as a model for increased severity of severe acute respiratory syndrome in elderly humans. J. Virol. 79, 5833-5838 (2005).

30. Kotenko, S. V. et al. IFN- $\lambda$ s mediate antiviral protection through a distinct class II cytokine receptor complex. Nat. Immunol. 4, 69-77 (2003).

31. Sheppard, P. et al. IL-28, IL-29 and their class II cytokine receptor IL-28R. Nat. Immunol. 4, 63-68 (2003).

32. Elazar, M. \& Glenn, J. S. Emerging concepts for the treatment of hepatitis delta. Curr. Opin. Virol. 24, 55-59 (2017).

33. Lan, J. et al. Structure of the SARS-CoV-2 spike receptor-binding domain bound to the ACE2 receptor. Nature $\mathbf{5 8 1}, 215-220$ (2020).

34. Shang, J. et al. Structural basis of receptor recognition by SARS-CoV-2. Nature 581, 221-224 (2020).

35. Felgenhauer, U. et al. Inhibition of SARS-CoV-2 by type I and type III interferons. J. Biol. Chem. jbc.AC120.013788 (2020).

36. Muir, A. J. et al. A randomized phase $2 b$ study of peginterferon lambda-1a for the treatment of chronic HCV infection. J. Hepatol. 61, 1238-1246 (2014).

37. Chan, H. L. Y. et al. Peginterferon lambda for the treatment of HBeAg-positive chronic hepatitis B: A randomized phase 2b study (LIRA-B). J. Hepatol. 64, 1011-1019 (2016).

38. Li, L. et al. IFN-lambda preferably inhibits PEDV infection of porcine intestinal epithelial cells compared with IFN-alpha. Antiviral Res. 140, 76-82 (2017).

39. Mordstein, M. et al. Lambda interferon renders epithelial cells of the respiratory and gastrointestinal tracts resistant to viral infections. J. Virol. 84, 5670-5677 (2010).

40. Rockx, B. et al. Escape from human monoclonal antibody neutralization affects in vitro and in vivo fitness of severe acute respiratory syndrome coronavirus. J. Infect. Dis. 201, 946-955 (2010).

41. Sui, J. et al. Effects of human anti-spike protein receptor binding domain antibodies on severe acute respiratory syndrome coronavirus neutralization escape and fitness. J. Virol. 88, 13769-13780 (2014)

42. Rockx, B. et al. Structural basis for potent cross-neutralizing human monoclonal antibody protection against lethal human and zoonotic severe acute respiratory syndrome coronavirus challenge. J. Virol. 82, 3220-3235 (2008).

43. Zost, S. J. et al. Potently neutralizing and protective human antibodies against SARS-CoV-2. Nature 584, 443-449 (2020).

44. McCray, P. B. Jr et al. Lethal infection of K18-hACE2 mice infected with severe acute respiratory syndrome coronavirus. J. Virol. 81, 813-821 (2007).

Publisher's note Springer Nature remains neutral with regard to jurisdictional claims in published maps and institutional affiliations.

(c) The Author(s), under exclusive licence to Springer Nature Limited 2020 


\section{Methods}

No statistical methods were used to predetermine sample size. The experiments were not randomized. The investigators were not blinded to allocation during experiments and outcome assessment.

\section{Ethics and biosafety}

The generation of recombinant SARS-CoV-2 MA was approved for use under BSL3 conditions by the University of North Carolina at Chapel Hill Institutional Review Board (UNC-CH IBC) and by a Potential Pandemic Pathogen Care and Oversight committee at the National Institute of Allergy and Infectious Diseases (NIAID). All animal work was approved by Institutional Animal Care and Use Committee at University of North Carolina at Chapel Hill according to guidelines outlined by the Association for the Assessment and Accreditation of Laboratory Animal Care and the US Department of Agriculture. All work was performed with approved standard operating procedures and safety conditions for SARS-CoV-2. Our institutional BSL3 facilities have been designed to conform to the safety requirements recommended by Biosafety in Microbiological and Biomedical Laboratories (BMBL), the US Department of Health and Human Services, the Public Health Service, the Centers for Disease Control and Prevention (CDC), and the National Institutes of Health (NIH). Laboratory safety plans have been submitted, and the facility has been approved for use by the UNC Department of Environmental Health and Safety (EHS) and the CDC.

\section{SARS-CoV-2 S RBD and ACE2 analysis and modelling}

Group 2B coronavirus $S$ and ACE2 amino acid sequences were aligned using Geneious Prime (v.2020.0.5). Accession numbers used were: SARS-CoV Urbani (AY278741), WIV1 (KF367457), SHC014 (KC881005), SARS-CoV-2 (MN985325.1), human ACE2 (BAB40370) and mouse ACE2 (NP_081562). Protein similarity scores were calculated using BLOSUM62 matrix. Contact residues previously identified by crystal structures ${ }^{33,34,45}$. Structure modelling was performed using Modeller (v.9.20) and visualized using PyMOL (v.1.8.6.0).

\section{Viruses, cells and transfections}

All viruses used were derived from an infectious clone of SARS-CoV-2, which was designed using similar strategies for SARS-CoV and MERS-CoV 22,46,47. The Q498Y/P499T substitutions were generated by site-directed mutagenesis using the following primers: forward: 5'-ATATGGTTTCTACACG ACTAATGGTGTTGGTTACCAACC-3' , reverse: 5'-TAGTCGTGTAGAAACCAT ATGATTGTAAAGGAAAGTAACAATT AAAACCTTC-3'. Viruses were derived following systematic cDNA assembly of the infectious clone, followed by in vitro transcription and electroporation into Vero E6 cells. Virus stocks were passaged once on Vero E6 cells and titrated via plaque assay. In brief, virus was serial diluted and inoculated onto confluent monolayers of Vero E6 cells, followed by agarose overlay. Plaques were visualized at 2 dpi by staining with neutral red dye.

Vero E6 cells were obtained from USAMRIID in 2003 (ATCC CRL-1586) and were maintained in Dulbecco's modified Eagle's medium (DMEM; Gibco), 5\% Fetal Clone II serum (FCII, Hyclone), and $1 \times$ antibiotic-antimycotic (Gibco). DBT-9 cells were previously clonally derived in our laboratory, maintained in DMEM, $10 \% \mathrm{FCII}$, and $1 \times$ antibiotic-antimycotic. Cells were confirmed to be negative for mycoplasma contamination.

For the Vero E6 single step growth curve, cells were infected at a multiplicity of infection (MOI) of 0.5 for $1 \mathrm{~h}$. Inoculum was removed and monolayer was washed twice with PBS and replaced with medium. For the HAE cell growth curve, cells were infected at an MOI of 0.5 for $2 \mathrm{~h}$. Inoculum was removed and cells were washed three times with PBS. At designated time points, Vero E6 supernatant was removed without replacement or HAE cells were washed apically with $200 \mu \mathrm{l}$ $1 \times$ PBS for $10 \mathrm{~min}$ and stored at $-80^{\circ} \mathrm{C}$ until titrated by plaque assay as described above.
For ACE2 receptor usage, non-permissive DBT-9 cells were transfected with pcDNA3.1 empty vector, pcDNA3.1-hACE2 or pcDNA3.1-mACE2 using Lipofectamine 2000 (Invitrogen). At $24 \mathrm{~h}$ after transfection, cells were infected at an MOI of 0.5 for $1 \mathrm{~h}$, removed and washed twice with PBS. At 24 h after infection, medium was removed, and total cellular RNA was collected via TRIzol (Invitrogen) and extracted using Direct-Zol RNA MiniPrep kit (Zymo Research). Viral RNA was quantified via qRT-PCR using TaqMan Fast Virus 1-Step Master Mix (Thermo Fisher Scientific) on a QuantStudio 3 (Applied Biosystems). SARS-CoV-2 RNA was quantified using US Centers of Disease Control and Prevention diagnostic N1 assay with the primers: forward: 5'-GACCCCAAAATCAGCGAAAT-3', probe: 5'-FAM-ACCCCGCATTACGTTTGGTGGAC C-BHQ1-3', reverse: 5'-TCTGGTTACTGCCAGTTGAATCTG-3'. Host $18 \mathrm{~S}$ rRNA was used as housekeeping control (Invitrogen, product number 4319413E). Viral RNA was analysed using the $\Delta \Delta C \mathrm{t}$ method and fold change over viral RNA in cell transfected with empty vector.

\section{In vivo Infections}

Hfh4-ACE2-overexpressing mice were bred and maintained at University of North Carolina at Chapel Hill. BALB/c mice were obtained from Envigo (strain 047). Mice were infected with $10^{5}$ PFU intranasally under ketamine-xylazine anaesthesia. Body weight was monitored daily and whole-body plethysmography (WBP) was performed as indicated. In brief, mice were allowed to equilibrate in WBP chambers (DSI Buxco respiratory solutions, DSI) for $30 \mathrm{~min}$ before a $5 \mathrm{~min}$ data acquisition period using FinePointe software. At indicated time points, a subset of mice were euthanized by isoflurane overdose and tissue samples were collected for titre and histopathology analysis. A subset of mice for nasal turbinate histopathology were perfused with $10 \%$ phosphate buffered formalin before tissue collection. The right caudal lung lobe was taken for titration and stored at $-80^{\circ} \mathrm{C}$ until homogenized in $1 \mathrm{ml}$ PBS and titrated by plaque assay as described above. The left lung lobe was taken for histopathology and were fixed in $10 \%$ phosphate buffered formalin for 7 days before paraffin embedding and sectioning.

\section{Histopathology and antigen staining}

Lungs were fixed for 7 days in $10 \%$ phosphate buffered formalin, paraffin embedded, and sectioned at $4 \mu \mathrm{m}$. Serial sections were stained with haematoxylin and eosin, and stained for immunohistochemistry for SARS-CoV-2 $\mathrm{N}$ using a monoclonal anti-SARS-CoV N antibody (1:250, NB100-56576, Novus Biologicals) on deparaffinized sections on the Ventana Discovery Ultra platform (Roche). Photomicrographs were captured on an Olympus BX43 light microscope at $200 \times$ magnification with a DP27 camera using cellSens Entry software.

\section{Vaccination studies}

Non-select BSL2 Venezuelan equine encephalitis virus strain 3526 based replicon particles (VRPs) were generated to express GFP, SARS-CoV-2S or $\mathrm{N}$ as previously described ${ }^{5,48}$. Mice were vaccinated via hind footpad infection with $10^{3} \mathrm{VRP}$ in $10 \mu \mathrm{l}$, boosted identically at 3 weeks post prime, and bled via submandibular bleed at 3 weeks to confirm presence of neutralizing antibodies. Neutralizing antibody levels were assessed via neutralization assay using wild-type SARS-CoV-2 expressing nanoluciferase (nLUC) in place of ORF7a. In brief, the ORF7a gene of SARS-CoV-2 was removed from the molecular clone and nLUC inserted downstream of the ORF7a transcription regulatory sequence. Recombinant viruses encoding nLUC (SARS-CoV- 2 nLUC) were recovered, titrated and serial dilutions of sera were incubated with virus for $1 \mathrm{~h}$ at $37^{\circ} \mathrm{C}$, then added to monolayers of Vero E6 cells. Forty-eight hours after infection, viral infection was quantified using nLUC activity via Nano-Glo Luciferase Assay System (Promega). IC $_{50}$ values were calculated from full dilution curves.

Mice were challenged 4 weeks post boost with $10^{5}$ PFU intranasally under ketamine-xylazine anaesthesia. Body weight was monitored daily. On day 2 after infection, mice were euthanized by isoflurane 
overdose and tissue samples were collected for titre analysis as described above.

\section{PEG-IFN- $\lambda 1$ treatment in vitro and in vivo}

PEG-IFN- $\lambda 1$ was obtained from Eiger BioPharmaceuticals by MTA in GMP prefilled syringes, $0.18 \mathrm{mg}$ per syringe $\left(0.4 \mathrm{mg} \mathrm{ml}^{-1}\right)$. Primary HAE cell cultures were obtained from the Tissue Procurement and Cell Culture Core Laboratory in the Marsico Lung Institute/Cystic Fibrosis Research Center at UNC. Human tracheobronchial epithelial cells provided by S. Randell were obtained from airway specimens resected from patients undergoing surgery under University of North Carolina Institutional Review Board-approved protocols (no. 03-1396) by the Cystic Fibrosis Center Tissue Culture Core. Primary cells were expanded to generate passage 1 cells and passage 2 cells were plated at a density of 250,000 cells per well on Transwell-COL (12-mm diameter) supports (Corning). HAE cell cultures were generated by differentiation at an air-liquid interface for 6 to 8 weeks to form well-differentiated, polarized cultures that resembled in vivo pseudostratified mucociliary epithelium ${ }^{49}$. HAE cells were treated with a range of PEG-IFN- $\lambda 1$ doses basolaterally for $24 \mathrm{~h}$ before infection. Remdesivir $(1 \mu \mathrm{M})$ was obtained from Gilead Sciences by MTA and was used as a positive control. Cultures were infected at an MOI of 0.5 for $2 \mathrm{~h}$. Inoculum was removed and culture was washed three times with PBS. At $48 \mathrm{~h}$ after infection, apical washes were taken to measure viral replication via plaque assays as described above. This study was repeated in two separate human donors.

One-year-old or 10-week-old BALB/c, or 4- to 7-week old Hfh4-ACE2 mice were subcutaneously treated with a single $2 \mu \mathrm{g}$ dose of PEG-IFN- $\lambda 1$ prophylactically at $18 \mathrm{~h}$ before infection, therapeutically at $12 \mathrm{~h}$ after infection $^{50}$, or PBS-vehicle-treated, and infected with $10^{5}$ PFU of SARS-CoV-2 MA intranasally under ketamine-xylazine anaesthesia. Hfh4-ACE2 mice at 4 to 7 weeks of age were treated as above and infected with $10^{5}$ PFU of SARS-CoV-2 MA. Body weight was monitored daily. WBP was performed as indicated. On days indicated, mice were euthanized by isoflurane overdose and tissue samples were collected for titre analysis as described above.

\section{Data analysis and presentation}

All data were visualized and analysed in Prism (v.8.4.2). Non-parametric tests were performed as described in figure legends. Figures were arranged in Adobe Illustrator (v.24.1).

\section{Reporting summary}

Further information on research design is available in the Nature Research Reporting Summary linked to this paper.

\section{Data availability}

All relevant data are included in this Article. Viral genome sequences have been uploaded to GenBank under accession numbers MT844088 (SARS-CoV-2 MA) and MT844089 (SARS-CoV-2 nLuc). Reagents and resources are available upon request from the corresponding author and with a material transfer agreement. Source data are provided with this paper.

45. Li, F., Li, W., Farzan, M. \& Harrison, S. C. Structure of SARS coronavirus spike receptor-binding domain complexed with receptor. Science 309, 1864-1868 (2005).

46. Yount, B. et al. Reverse genetics with a full-length infectious cDNA of severe acute respiratory syndrome coronavirus. Proc. Natl Acad. Sci. USA 100, 12995-13000 (2003)

47. Scobey, T. et al. Reverse genetics with a full-length infectious cDNA of the Middle East respiratory syndrome coronavirus. Proc. Natl Acad. Sci. USA 110, 16157-16162 (2013).

48. Agnihothram, S. et al. Development of a broadly accessible Venezuelan equine encephalitis virus replicon particle vaccine platform. J. Virol. 92, e00027-18 (2018).

49. Fulcher, M. L., Gabriel, S., Burns, K. A., Yankaskas, J. R. \& Randell, S. H. Well-differentiated human airway epithelial cell cultures. Methods Mol. Med. 107, 183-206 (2005).

50. Davidson, S. et al. IFN $\lambda$ is a potent anti-influenza therapeutic without the inflammatory side effects of IFNa treatment. EMBO Mol. Med. 8, 1099-1112 (2016).

Acknowledgements This project was funded in part by the National Institute of Allergy and Infectious Diseases, National Institutes of Health, Department of Health and Human Service award: 1 U19 Al142759 (Antiviral Drug Discovery and Development Center awarded to R.S.B.); 5R01A1132178 (partnership grant awarded to T.P.S. and R.S.B.) and an animal models contract from the NIH (HHSN272201700036I). This project was supported in part by the North Carolina Policy Collaboratory at the University of North Carolina at Chapel Hill with funding from the North Carolina Coronavirus Relief Fund established and appropriated by the North Carolina General Assembly. K.H.D. is funded by an NIH NIAID T32 AIO07419. D.R.M. is funded by an NIH NIAID T32 AI007151 and a Burroughs Wellcome Fund Postdoctoral Enrichment Program Award. The Marsico Lung Institute Tissue Procurement and Cell Culture Core is supported by NIH grant DK065988 and Cystic Fibrosis Foundation grant BOUCHE15RO. Animal histopathology service was performed by D. Hilliard and L. Wang in the Animal Histopathology and Laboratory Medicine Core at the University of North Carolina, which is supported in part by an $\mathrm{NCl}$ Center Core Support Grant (5P30CA016086-41) to the UNC Lineberger Comprehensive Cancer Center.

Author contributions K.H.D. and S.R.L. designed and conducted in vitro and animal experiments, analysed data, generated figures and wrote the manuscript. A.S. conducted animal experiments, C.E.E. generated VRP vaccine and vaccinated mice. D.R.M. conducted the neutralization assay and analysed data. S.A.M. imaged and analysed histology. A.W., K.L.G. and A.J.B. assisted with animal experiments. B.L.Y. and Y.J.H. performed cloning. L.E.A. and E.H. assisted with transfection experiments. M.D.B., I.C.C. and J.S.G. provided resources and helped to design and analyse experiments with PEG-IFN- $\lambda$ 1. L.E.G conducted experiments, analysed data and edited the manuscript. T.P.S. conducted experiments, analysed data and wrote the manuscript. R.S.B. supervised the project and wrote the manuscript.

Competing interests M.D.B and I.C.C. are employees, and J.S.G is the founder and a board member of, Eiger BioPharmaceuticals, Inc., which produces peg-IFN- $\lambda 1$.

Additional information

Supplementary information is available for this paper at https://doi.org/10.1038/s41586-020 2708-8.

Correspondence and requests for materials should be addressed to R.S.B.

Peer review information Nature thanks Emmie de Wit and the other, anonymous, reviewer(s) for their contribution to the peer review of this work.

Reprints and permissions information is available at http://www.nature.com/reprints. 
A

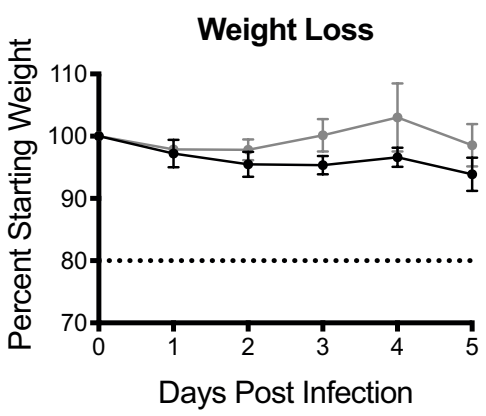

D

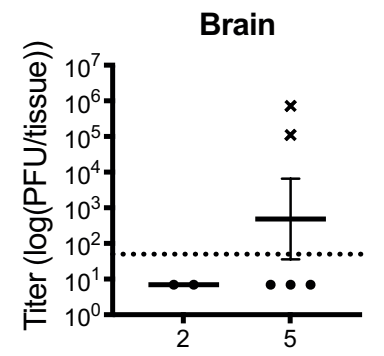

Days Post Infection
B

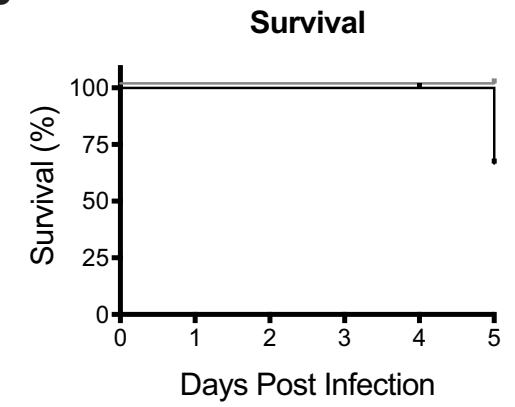

$\mathbf{E}$

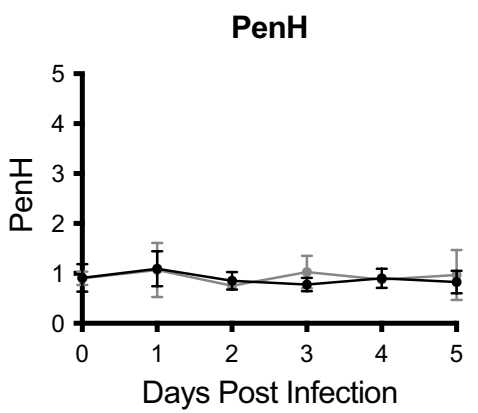

C

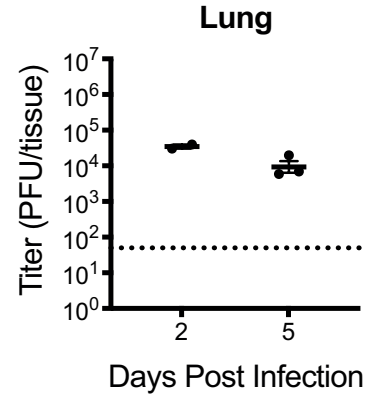

$\mathbf{F}$

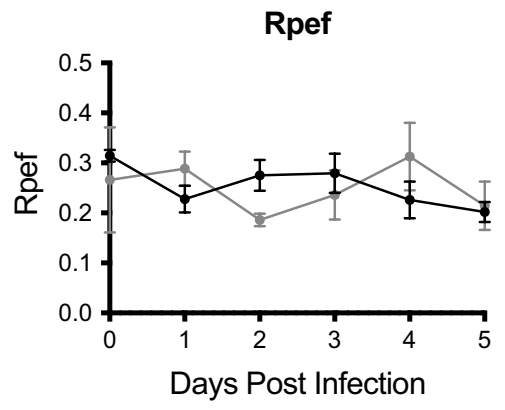

Extended Data Fig. 1 | SARS-CoV-2 infection in Hfh4-hACE2 transgenic mice. a-f, Male and female $H f h 4-h A C E 2$ mice were infected with $10^{5}$ PFU SARS-CoV-2 WT. a, Per cent starting weight. Dotted line represents weight-loss criteria for humane euthanasia. $n=2$ mock and 5 SARS-CoV-2. b, Survival. c, d, Lung (c) and brain (d) viral titre. Dotted line represents LOD. Undetected samples are plotted at half the LOD. ' $x$ ' symbol indicates mice that succumbed to infection. $2 \mathrm{dpi}: n=2 ; 5 \mathrm{dpi}: n=5$.e,f, Whole body plethysmography assessing pulmonary function for PenH (e) and Rpef (f). 
Consensus

SARS-CoV-1 Urbani

SARS-CoV-1 MA15

WIV1

SHC014

SARS-COV-2

Consensus

SARS-CoV-1 Urbani

SARS-CoV-1 MA15

WIV1

SHC014

SARS-CoV-2

\section{Consensus \\ SARS-CoV-1 Urbani \\ SARS-CoV-1 MA15 \\ WIV1 \\ SHC014 \\ SARS-CoV-2}

Consensus

SARS-CoV-1 Urbani

SARS-COV-1 MA15

WIV1

SHC014

SARS-CoV-2
NITNLCPFGE VFNATXFXSV YAWXRKXISN CVADYSVLYN SXXFSTFKCY

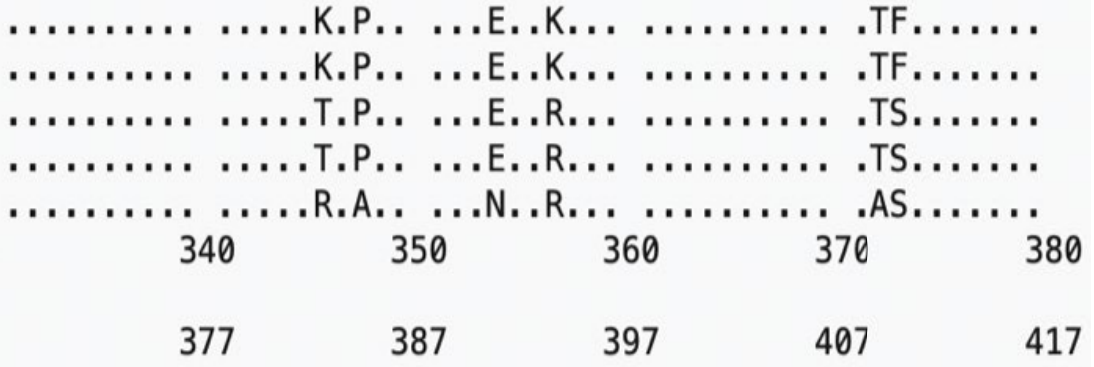

GVSXTKLNDL CFXNVYADSF VXXGDXVRQI APGQTGXIAD YNYKLPDDFX

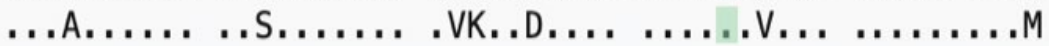

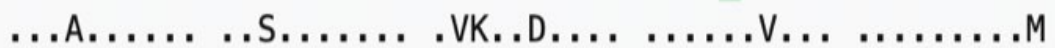

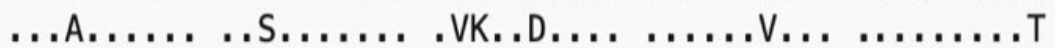

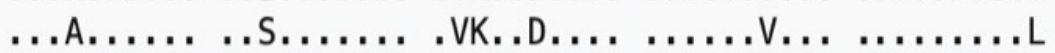

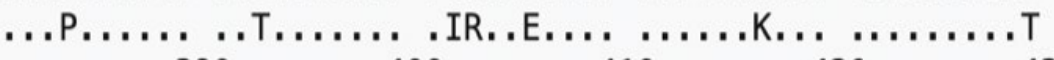
$\begin{array}{lllll}390 & 400 & 410 & 420 & 430\end{array}$

$\begin{array}{lllll}427 & 437 & 447 & 457 & 467\end{array}$

GCVJAWNXXX XDXXXXGNXN YXYRXXRXXX LXPXERDJSX XXXXXXXXXC ...L...TRN I.ATST..Y. .K..YL.HGK .R.F...I.N VPFSPDGKP. ...L...TRN I.ATST..H. .K..YL.HGK .R.F...I.N VPFSPDGKP. ...L...TRN I.ATQT..Y. .K..SL.HGK .R.F...I.N VPFSPDGKP. ...L...TNS K.SSTS..Y. .L..WV.RSK .N.Y...L.N DIYSPGGQS. ...I...SNN L.SKVG..Y. .L..LF.KSN .K.F...I.T EIYQAGSTP. $\begin{array}{lllll}440 & 450 & 460 & 470 & 480\end{array}$

$476 \quad 486 \quad 496 \quad 506$

XXXXXXNCYX PLXXYGFXXT XGXGXQPYRV VVLSFELLXA PATVCGP TPP-AL...W ...ND...YT, T.I.Y.... ........ ...... TPP-AL...W ..ND...YT. T.I.Y.... ........ ......

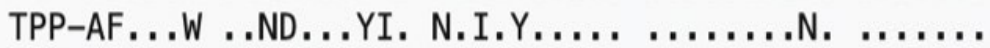
SAV-GP...N ..RP...FT. A.V.H.... ................ NGVEGF...F . .QS...QP. N.V.Y.................. $490 \quad * 500 \quad 510 \quad 520$

Extended Data Fig. 2 | Group 2B coronavirus spike RBD alignment. Amino acid positions are numbered above in reference to SARS-CoV-1, and below in reference to SARS-CoV-2. Green highlighted residues are hACE2 contacts as determined by published crystal structures. 

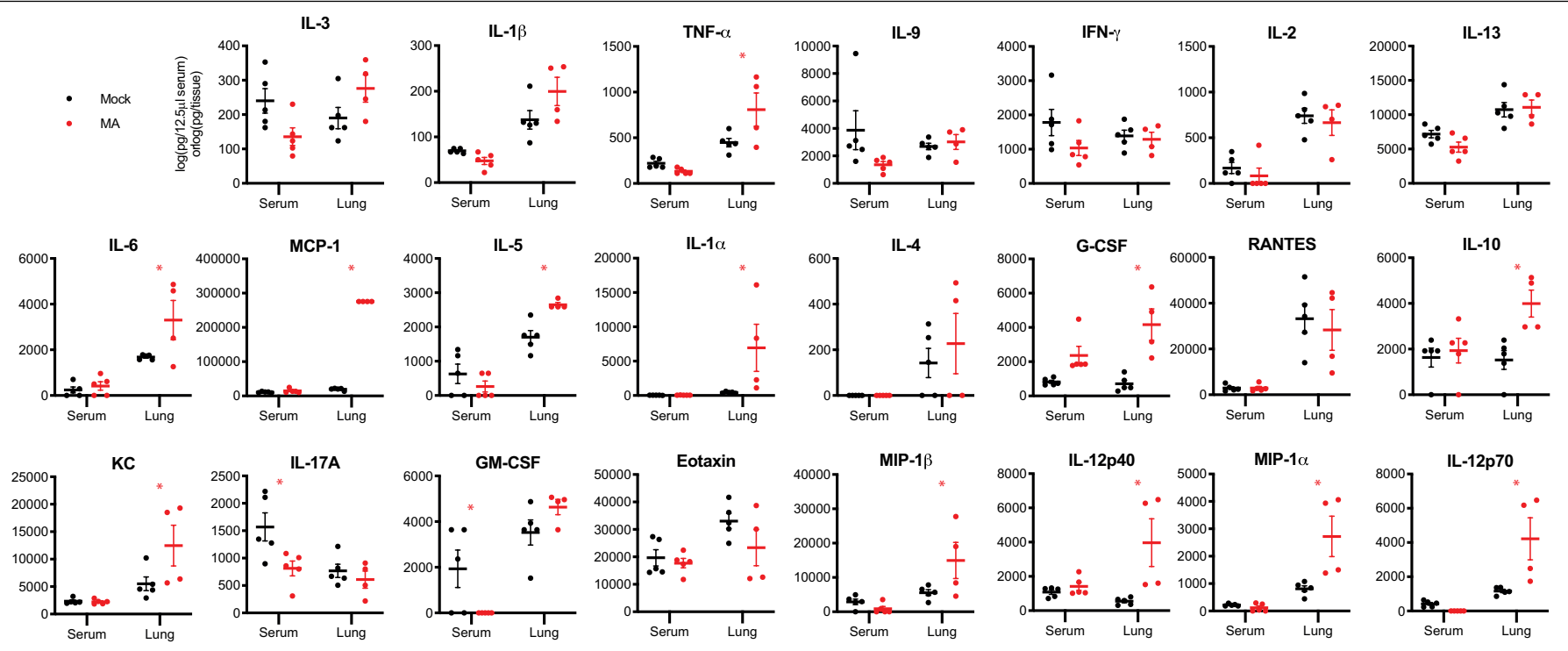

Extended Data Fig. 3 | Cytokine analysis in SARS-CoV-2 MA infected 1-year-old BALB/c mice. Cytokine and chemokine levels in serum and lung homogenates of 1-year-old female BALB/c mice from Fig. 3 at 2 and 4 dpi. For each analyte, data analysed by 2-way ANOVA followed by Sidak's multiple comparisons. ${ }^{* \prime}$ denotes $P=0.0155$ (TNF- $\alpha$ ), 0.0189 (IL-6), $<0.0001$ (MCP-1), 0.0115 (IL-5), 0.0127 (IL-1 $\alpha$ ), 0.0004 (G-CSF), 0.0070 (IL-10), 0.0243 (KC), 0.0152 (IL-17A), 0.0408 (GM-CSF), 0.0261 (MIP-1 $\beta$ ), 0.0025 (IL-12p40), 0.0015 (MIP-1 $\alpha$ ), 0.0019 (IL-12p70). 
A

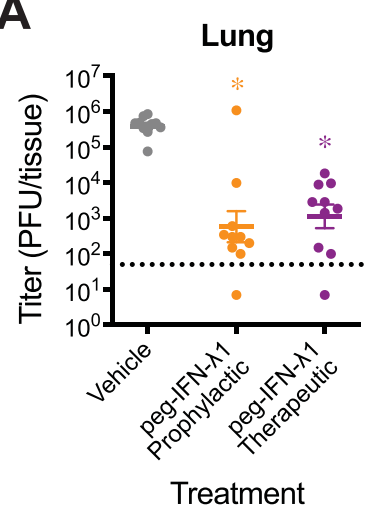

C

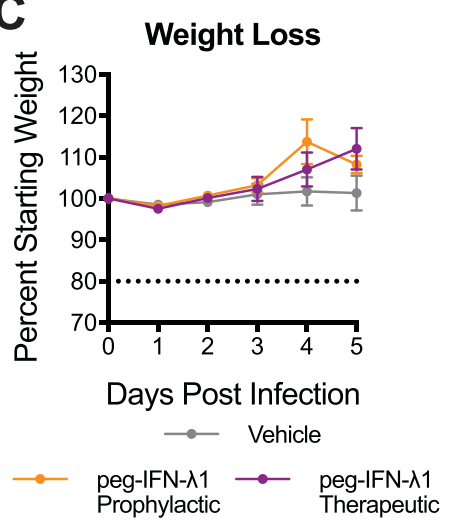

Extended Data Fig. 4 | Evaluation of peg-IFN- $\lambda 1$ against SARS-CoV-2 MA infection in young BALB/c and Hfh4-hACE2 mice. a, b, Twelve-week-old female BALB/c mice were subcutaneously treated with vehicle or with $2 \mu \mathrm{g}$ peg-IFN- $\lambda 1$ prophylactically or therapeutically and infected with SARS-CoV-2 MA. Viral titres in the lung (a) and nasal turbinates (b) at $2 \mathrm{dpi} . n=10$ for each group, combined from two independent experiments. Dotted line represents LOD. Undetected samples plotted at half the LOD. log-transformed data analysed by 1-way ANOVA followed by Holm-Sidak's multiple comparisons. In $\mathbf{a},{ }^{\prime \prime \prime}$ denotes $P=<0.0001$ (prophylactic), $<0.0001$ (therapeutic). $\mathbf{c}-\mathbf{e}$, Four-to seven-week-old $H f h 4$ - $h A C E 2$ male and female mice were treated with peg-IFN- $\lambda 1$ as done in $\mathbf{a}, \mathbf{b}$, and infected with $10^{5}$ PFU SARS-CoV- 2 WT. $n=8$ vehicle; $n=10$ prohpylactic, $n=7$ therapeutic. , Per cent starting weight. Dotted line represents weight-loss criteria for humane euthanasia. Data analysed by mixed effects analysis followed by Sidak's multiple comparisons.d, Survival. e, Lung viral titre at 2 and $5 \mathrm{dpi}$. $2 \mathrm{dpi}: n=4$ vehicle, $n=4$ prophylactic, $n=4$ therapeutic: $5 \mathrm{dpi}: n=3$ vehicle, $n=4$ prophylactic, $n=2$. Data analysed by two-way ANOVA followed by Dunnett's multiple comparisons. ${ }^{*{ }^{\prime}}$ denotes $P=0.0037$ (prophylactic, $2 \mathrm{dpi}$ ), 0.0365 (therapeutic, $2 \mathrm{dpi}$ ). All error bars represent standard error about the mean. 


\section{Reporting Summary}

Nature Research wishes to improve the reproducibility of the work that we publish. This form provides structure for consistency and transparency in reporting. For further information on Nature Research policies, see our Editorial Policies and the Editorial Policy Checklist.

\section{Statistics}

For all statistical analyses, confirm that the following items are present in the figure legend, table legend, main text, or Methods section.

n/a Confirmed

$\square$ The exact sample size $(n)$ for each experimental group/condition, given as a discrete number and unit of measurement

$\square$ \A statement on whether measurements were taken from distinct samples or whether the same sample was measured repeatedly

$\square$ The statistical test(s) used AND whether they are one- or two-sided

$\square$ Only common tests should be described solely by name; describe more complex techniques in the Methods section.

Х $\square$ A description of all covariates tested

$\square$ \A description of any assumptions or corrections, such as tests of normality and adjustment for multiple comparisons

$\square$ A full description of the statistical parameters including central tendency (e.g. means) or other basic estimates (e.g. regression coefficient)

$\bigotimes$ AND variation (e.g. standard deviation) or associated estimates of uncertainty (e.g. confidence intervals)

$\varnothing$ For null hypothesis testing, the test statistic (e.g. $F, t, r$ ) with confidence intervals, effect sizes, degrees of freedom and $P$ value noted

Give $P$ values as exact values whenever suitable.

\ $\square$ For Bayesian analysis, information on the choice of priors and Markov chain Monte Carlo settings

Х $\square$ For hierarchical and complex designs, identification of the appropriate level for tests and full reporting of outcomes

Х $\square$ Estimates of effect sizes (e.g. Cohen's $d$, Pearson's $r$ ), indicating how they were calculated

Our web collection on statistics for biologists contains articles on many of the points above.

\section{Software and code}

Policy information about availability of computer code

Data collection WBP data was collected with FinePointe software (Version 2.3.1.16). Images captured with cellSense Entry software (Olympus, Version 3.1 qRT-PCR data was collected using Design \& Analysis software (ThermoFisher, version 2.4)

Data analysis Geneious Prime (Version 2020.0.5), Modeller (Version 9.20), PyMOL (Version 1.8.6.0), Prism (Version 8.4.2)

For manuscripts utilizing custom algorithms or software that are central to the research but not yet described in published literature, software must be made available to editors and reviewers. We strongly encourage code deposition in a community repository (e.g. GitHub). See the Nature Research guidelines for submitting code \& software for further information.

\section{Data}

Policy information about availability of data

All manuscripts must include a data availability statement. This statement should provide the following information, where applicable:

- Accession codes, unique identifiers, or web links for publicly available datasets

- A list of figures that have associated raw data

- A description of any restrictions on data availability

All relevant data is included in this article. Viral genome sequences uploaded to GenBank (SARS-CoV-2 MA: MT844088. SARS-CoV-2 nLuc: MT844089). Reagents and resources available upon request to corresponding author (rbaric@email.unc.edu) and with material transfer agreement. 


\section{Field-specific reporting}

Please select the one below that is the best fit for your research. If you are not sure, read the appropriate sections before making your selection. \Life sciences

\section{Life sciences study design}

All studies must disclose on these points even when the disclosure is negative.

$\begin{array}{ll}\text { Sample size } & \text { Sample size calculations were not performed. Sample sizes were chosen based on previous experience with mouse models of coronavirus } \\ \text { infection which show that use of 5-10 mice per group are sufficient for statistical power of over } 80 \% \text {. Fo ascertain reproducibility, each }\end{array}$ experiment was performed at least twice, each with sufficient sample sizes to calculate statistics.

Data exclusions No data was excluded from this manuscript

Replication All experiments were conducted at least twice and were successfully reproduced.

Randomization Mice were assigned to experimental groups randomly. For experiments other than animal studies, randomization was not relevant as this is a controlled observational study.

Blinding Investigators were not blinded except for lung pathology evaluation. Other assays measure quantitative unbiased data.

\section{Reporting for specific materials, systems and methods}

We require information from authors about some types of materials, experimental systems and methods used in many studies. Here, indicate whether each material, system or method listed is relevant to your study. If you are not sure if a list item applies to your research, read the appropriate section before selecting a response.

Materials \& experimental systems

\begin{tabular}{l|l}
\hline$n / a$ & Involved in the study \\
\hline & $\bigotimes$ Antibodies \\
$\square$ & $\square$ Eukaryotic cell lines \\
$\square$ & $\square$ Animals and other organisms \\
$\square$ & $\square$ Clinical data \\
$\square$ & $\square$ Dual use research of concern
\end{tabular}

Methods

n/a $\quad$ Involved in the study

\ $\square$ ChIP-seq

Х Flow cytometry

\ $\square$ MRI-based neuroimaging

\section{Antibodies}

Antibodies used

Validation
SARS nucleocapsid protein (NB100-56576, Novus Biologicals) used at 1:250 dilution on Ventana Discovery Ultra platform (Roche).

This antibody has been used widely in studies from our lab with high signal and low background in immunohistochemistry. See DOI: 10.1126/scitransImed.aal3653.

\section{Eukaryotic cell lines}

\section{Policy information about cell lines}

Cell line source(s)

Authentication

Mycoplasma contamination

Commonly misidentified lines (See ICLAC register)
Vero E6 cells obtained from USAMRIID in 2003. Original source: ATCC CRL-1586. DBT-9 cells derived in the Baric Lab (DOI: 10.1006/viro.1996.8402)

Cell lines were not authenticated

Vero E6 cells have been confirmed to be mycoplasma free by PCR based assay

None 


\section{Animals and other organisms}

Policy information about studies involving animals; ARRIVE guidelines recommended for reporting animal research

Laboratory animals

12-week and 1-year old female BALB/c mice were obtained from Envigo. hACE2 transgenic mice were derived in our lab and maintained at UNC at Chapel Hill. Animals were housed up to 5 mice per cage at $18-24^{\circ} \mathrm{C}$ on $12 \mathrm{hr}$ light/dark cycle. Food and water were provided ad libitum.

Wild animals

This study did not involve wild animals

Field-collected samples

This study did not involve field-collected samples

Ethics oversight

The generation of recombinant SARS-CoV-2 MA was approved for use under BSL3 conditions by the University of North Carolina at Chapel Hill Institutional Review Board (UNC-CH IBC) and by a Potential Pandemic Pathogen Care and Oversight committee at the National Institute of Allergy and Infectious Diseases (NIAID). All animal work was approved by Institutional Animal Care and Use Committee at University of North Carolina at Chapel Hill according to guidelines outlined by the Association for the Assessment and Accreditation of Laboratory Animal Care and the U.S. Department of Agriculture. All work was performed with approved standard operating procedures and safety conditions for SARS-CoV-2. Our institutional BSL3 facilities have been designed to conform to the safety requirements recommended by Biosafety in Microbiological and Biomedical Laboratories (BMBL), the U.S. Department of Health and Human Services, the Public Health Service, the Centers for Disease Control and Prevention (CDC), and the National Institutes of Health (NIH). Laboratory safety plans have been submitted, and the facility has been approved for use by the UNC Department of Environmental Health and Safety (EHS) and the CDC.

Note that full information on the approval of the study protocol must also be provided in the manuscript. 